\title{
Características sociodemográficas de estudiantes adolescentes con violencia familiar y bajo rendimiento académico, de una institución, Montería 2018
}

\section{Sociodemographic characteristics of adolescent students with family violence and low academic performance, from an institution, Montería 2018}

\author{
Cleiver Orozco $\mathrm{G}^{1}{ }^{\circledR}$, Regina Cogollo $\mathrm{J}^{2}$, Yuleidis Castellón $\mathrm{C}^{3}$
}

\begin{abstract}
RESUMEN
La violencia es reconocida como un problema de Salud Pública. De acuerdo con lo definido por la OMS y OPS, es uno de los principales factores que contribuyen a la muerte, la enfermedad y la discapacidad. Objetivo. Determinar las características sociodemográficas de estudiantes de secundaria de una institución académica de Montería con bajo rendimiento académico y violencia familiar. Materiales y métodos. Descriptivo, transversal, con enfoque cuantitativo. La muestra fue de 45 estudiantes entre 14 y 18 años, que presentaron bajo rendimiento académico. Resultados. En los estudiantes adolescentes predomina la edad de 14 años $(42 \%)$, el género masculino $(60 \%)$, octavo grado con una incidencia de $60 \%$. Predomina la violencia física con un $44 \%$, seguida de la violencia psicológica con un 33\%; el 38\% siempre tienen dificultades en su rendimiento académico. Conclusiones. La mayoría de los estudiantes adolescentes están en el grupo de edad de 14 años (adolescencia media), género masculino, estudian octavo grado y siempre tienen dificultades en su rendimiento académico. Un porcentaje significativo de la población estudiada presentó violencia física y psicológica, caracterizándose por golpes e insultos, siendo necesario intervenciones a nivel familiar donde se promueva principios y valores.
\end{abstract}

Palabras clave: violencia, adolescencia, rendimiento académico.

\begin{abstract}
Violence is recognized as a Public Health problem. As defined by the $\mathrm{WHO}$ and $\mathrm{PAHO}$, it is one of the main factors that contribute to death, disease, and disability. Objective. To determine the sociodemographic characteristics of high school students from an academic institution in Montería with low academic performance and family violence. Materials and methods. Descriptive, cross-sectional, with a quantitative approach. The sample was 45 students between 14 and 18 years old, who presented low academic performance. Results. The age of 14 years $(42 \%)$ predominates (middle adolescence), the male gender $(60 \%)$, they are in the eighth grade $(60 \%)$. Physical violence predominates with $44 \%$, followed by psychological violence with $33 \% ; 38 \%$ always have difficulties in their academic performance. Conclusions. Most of the adolescent students are in the age group of 14 years (middle adolescence), male, study eighth grade and always have difficulties in their academic performance. A significant percentage presented physical and psychological violence, characterized by beatings and insults, requiring interventions at the family level where principles and values are promoted.
\end{abstract}

Keywords: violence, adolescence, academic performance.
Citación (Vancouver)

Características sociodemográficas de estudiantes adolescentes con violencia familiar y bajo rendimiento académico, de una institución, Montería 2018 . Rev Avances en Salud; 2021. (5)1:41-47 e. doi: 10.21897/25394622.2597

(C) 2020. Universidad de Cordoba. Este es un artículo de acceso abierto distribuido bajo los términos de la licencia Creative Commons Attribution License, que permite el uso ilimitado, distribución y reproducción en cualquier medio, siempre que el autor original y la fuente se acreditan.

'Maestrante en Salud Pública. Enfermero. Universidad de Córdoba. cleiver_cjog@hotmail.com

${ }^{2}$ Esp. Salud Familiar. Mgt. en Enfermería, énfasis en Salud Familiar. Enfermera. Universidad de Córdoba. recogojim@hotmail.com ${ }^{3}$ Enfermera. Universidad de Córdoba. yuleidis98@hotmail.com 


\section{INTRODUCCIÓN}

La violencia es reconocida como un problema de salud pública $(1,2,3)$. De acuerdo con lo definido por la OMS y OPS (4), es uno de los principales factores que contribuyen a la muerte, la enfermedad y la discapacidad. La violencia, se puede encontrar de diferentes formas, la UNICEF (5) la clasifica como, violencia de género, violencia contra niños y niñas, violencia en la adolescencia, violencia contra la mujer y violencia familiar. Cabe resaltar que la violencia familiar afecta el desempeño social y psicológico de los integrantes de la familia en todos los ámbitos de su vida, dificultando el aprendizaje y socialización de la familia, en especial de los que estudian afectando su calidad de vida (6).

La violencia familiar, es un fenómeno que crece con el pasar de los días, de acuerdo con el Informe del Observatorio de Delito de la Gobernación de Córdoba (7), en el año 2017, el $30 \%$ de los casos reportados por violencias, son de tipo familiar. Cuando en un hogar se presenta violencia de cualquier tipo, los más afectados son los menores de edad, el cual se ve reflejada en actitudes negativas, generando bajo rendimiento académico (8).

La violencia familiar es una de las causas principales que influye en el rendimiento académico de los adolescentes, teniendo en cuenta la forma en que han vivido esta experiencia, dificulta el desarrollo óptimo para su personalidad, expresando necesidades de ayuda, seguridad, protección, amor y comprensión, ante un fenómeno que impacta en su vida cotidiana, dejando huellas difíciles de borrar que evidentemente afectan al adolescente (9). Por lo tanto, los adolescentes que han sido maltratados, necesitan ayudan psicológica para que este problema al que están siendo expuestos no afecte sus relaciones personales y desempeño académico (10).

La influencia de la violencia familiar en el bajo rendimiento académico, se puede expresar en diferentes formas, dependiendo de la capacidad que tenga la familia para abordar estos problemas, un estudio en donde se analizó la incidencia de la violencia familiar en estudiantes adolescentes de educación secundaria, concluye que la violencia psicológica y verbal son las más frecuentes en esta problemática, siendo el adolescente el principal agredido dentro del núcleo familiar, al igual, estos adolescentes se encuentran entre 14 y 16 años de edad (11).

El bajo rendimiento académico no es un estado permanente y depende tanto del individuo como del entorno familiar, López y otros (12), idealizan que es un problema que preocupa a padres y maestros, pues psicológicamente puede dejar secuelas en la vida, caracterizado por notas bajas y logros no alcanzado dentro de los rangos normales de las calificaciones.

Es por esto que el grupo investigador tiene como objetivo determinar las características sociodemográficas de estudiantes de secundaria de una institución académica de Montería con bajo rendimiento académico y violencia familiar en 2018.

\section{MATERIALES Y MÉTODOS}

Estudio transversal con enfoque cuantitativo. La población de este estudio estuvo conformada por 45 estudiantes de edades entre 14 y 18 años con bajo rendimiento académico, que asistían oficialmente a una Institución Educativa pública de Montería. La selección de la muestra fue por muestreo no probabilístico, seleccionando a los estudiantes que cumplieron con los criterios de inclusión.

\section{Criterios de inclusión}

- $\quad$ Ser estudiantes activos de la Institución Educativa IE pública seleccionada.

- Tener edades entre 14 y 18 años.

- Tener bajo rendimiento académico.

- Tener comportamientos de disciplina inadecuados en la institución.

- $\quad$ Participación voluntaria.

- $\quad$ Firmar el consentimiento informado por sus padres y/o acudientes. 


\section{Criterios de exclusión}

- Ser estudiantes destacados y con rendimiento académico adecuado.

- No tener permiso otorgado por los padres para el estudio de la investigación.

\section{Instrumento}

La recolección de datos se realizó mediante Encuesta diseñada por los investigadores, previa prueba piloto y aval de la Institución Educativa IE pública para realizar el estudio, alcanzando los objetivos propuestos en la investigación, donde se indagaron los datos sociodemográficos (edad, género, grado académico) de los estudiantes adolescentes al igual que el tipo de violencia que presentaron en sus hogares.

\section{Aspectos éticos y legales}

Los aspectos que se tuvieron en cuenta para el desarrollo legal de esta investigación, estuvo basado en la Resolución 8430 de 1993 (13), por la cual se establecen las normas científicas, técnicas y administrativas para la investigación en salud, en especial el Articulo 5, donde se estipula que en toda investigación donde el ser humano sea sujeto de estudio, deberá prevalecer el criterio de protección de sus derechos y el Articulo 11, en la cual se clasifica ésta población objeto en riesgo bajo, ya que los investigadores no realizaran procedimientos invasivos que afecte su integridad, física, biológica o psicológica.

\section{RESULTADOS}

Se analizó la caracterización sociodemográfica y el tipo de violencia familiar.

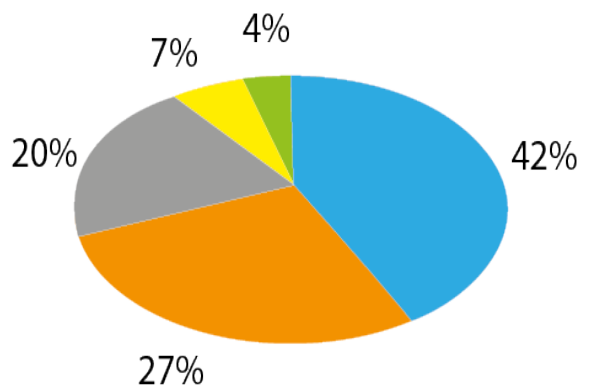

- 14 años 15 años $\square 16$ años $\square 17$ años $\square 18$ años

Gráfica 1. Edad de los Estudiantes adolescentes con bajo rendimiento académico.

En la gráfica 1 se observa que la mayoría de los adolescentes estuvieron en el grupo de edad de 14 años (42\%) con bajo rendimiento académico, situándose en la adolescencia media, en donde se caracterizan por el distanciamiento hacia los padres.

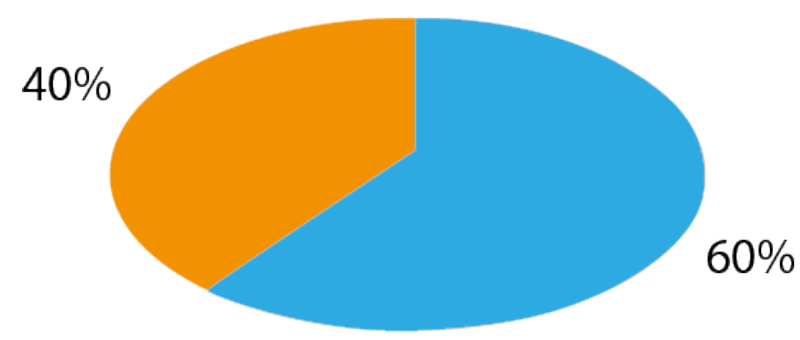

- Masculino $\quad$ Femenino

Gráfica 2. Género de los estudiantes adolescentes con bajo rendimiento académico.

En la clasificación por género de los estudiantes adolescentes, en la gráfica 2 se muestra que el género masculino $(60 \%)$ fue el que predominó en el estudio. Por lo que es oportuno desarrollar intervenciones que propendan al mejoramiento de su ámbito familiar y académico. 


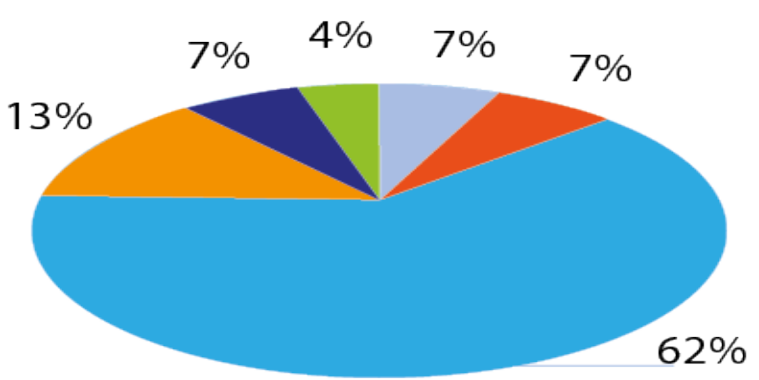

$\square 6^{\circ}=7^{\circ}=8^{\circ}=9^{\circ} \square 10^{\circ}=11^{\circ}$

Gráfica 3. Grado de los estudiantes adolescentes con bajo rendimiento académico.

En cuanto al grado académico de los estudiantes, se pudo notar que el más prevalente es el octavo grado con una incidencia de $60 \%$, y en menor proporción grado once con 4\%, donde prevalece el bajo rendimiento académico debido a diferentes conductas negativas en el estudiante adolescente generado por la violencia familiar (ver gráfica 3).

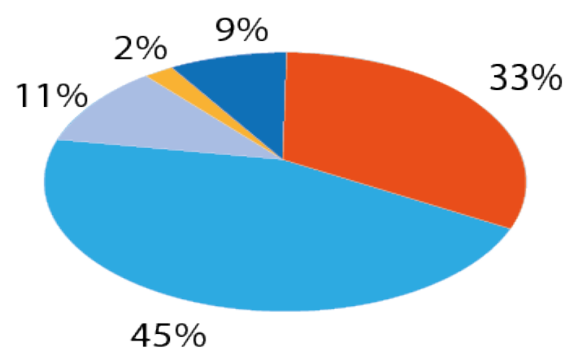

\section{Psicologia Fisica \\ Sexual Ninguna}

Gráfica 4. Tipo de violencia familiar en los estudiantes adolescentes con bajo rendimiento académico.

En los tipos de violencia familiar, los resultados muestran que la violencia física fue la más común con un 44\%, seguida de la violencia psicológica con un 33\%, y en menor medida violencia sexual con un $2 \%$, indicando que la incidencia de éste fenómeno es un desencadenante principal para que los estudiantes presenten las conductas inadecuadas en sus estudios, reflejándose en su bajo rendimiento académico, caracterizándose por golpes e insultos respectivamente, como lo estipula la UNICEF (ver gráfica 4).

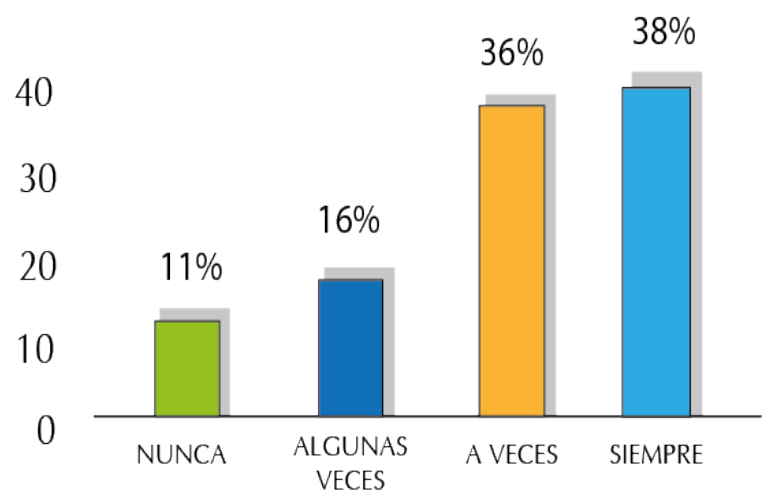

Gráfica 5. Dificultad en el rendimiento académico en los estudiantes adolescentes.

En la gráfica se observa que la mayoría de los estudiantes en $38 \%$, siempre tienen dificultades en su rendimiento académico, cuando presentan violencia familiar.

\section{DISCUSIÓN}

Estosresultados sonequivalentesa los concluidos por Gonzaga (14), quien concluye que la violencia familiar incide sobre las condiciones de vida de los adolescentes, y más en la etapa de la adolescencia media, donde se tornan vulnerables sus posibilidades de crecimiento y desarrollo y formación académica, quien se ve influenciada de forma negativa por este fenómeno. Asimismo, en esta etapa se torna más difícil el acceso a los servicios preventivos en salud, como concluyen Berrocal et al. (15), en su estudio realizado sobre la accesibilidad a los servicios preventivos, encontraron que el carecer de información precisa sobre derechos, deberes, y oferta de servicios de salud no fomenta el interés por participar de actividades de promoción de la salud y prevención de la enfermedad y más cuanto se presenta violencia familiar, quien puede afectar todas las áreas del adolescente. 
Asimismo, los resultados de la investigación son similares a los encontrados en el estudio realizado por Cano (16), donde este fenómeno, es más evidente en varones debido al Dentro de este ciclo del fenómeno como lo es la violencia familiar, los adolescentes son impactados de forma de negativa, reflejándose en todas las áreas de su vida, y más en lo académico.

Los adolescentes sufren si son víctima de la violencia familiar, además no tienen el apoyo de alguien de su familia y muchas veces las consecuencias de la violencia del maltrato por negligencia o descuido es la falta de atención de sus padres, no se les prepara comida, no se les lava y no se les cuida, reaccionando de forma agresiva en el ámbito escolar (20).

\section{CONCLUSIONES}

Se identificó que la mayoría, cursan octavo y noveno grado y presentan bajo rendimiento académico. Asimismo, un porcentaje significativo de la población estudiada presentó violencia física y psicológica, caracterizándose por golpes e insultos, siendo necesario intervenciones a nivel familiar donde se promueva principios y valores. En menor porcentaje se presentaron casos de violencia sexual dentro de la familia, lo que indica que los adolescentes y su familia necesitan una orientación oportuna por parte del personal adecuado, a fin de buscar soluciones a éste problema importante.

Finalmente, se evidencia que los adolescentes estudiantes no tienen orientación y asesoramiento educativo y legal por parte del área de orientación estudiantil de la institución educativa, quien no cumple con su rol fundamental de velar por el bienestar de los adolescentes, igualmente no se les brinda una valoración médica y psicológica para determinar su estado de salud tanto físico como psicológico, con la finalidad de identificar un posible maltrato para solicitar la respectiva indagación ante los organismos competentes.

\section{RECOMENDACIONES}

Motivar a los estudiantes a dirigirse a entidades como psicorientadores académicos, departamento estudiantil, docentes, para que sean orientados de forma idónea a entidades como Fiscalía, Juzgado de la Niñez y Adolescencia, cuando sean víctimas de violencia familiar en todas sus manifestaciones, sean estás físicas, psicológicas y sexuales ejercida por cualquier familiar o persona particular, con el fin de buscar solución a la problemática.

Fomentar y fortalecer la autoestima de los estudiantes víctimas de violencia familiar para que sean personas proactivas, conscientes de la realidad social en la que viven, incentivar la asistencia puntuala clases, la participación activa en las acciones académicas y el mejoramiento de su comportamiento proactivo dentro de la comunidad educativa y con sus familias, para que sean talentos que sirvan para una nueva sociedad llena de valores y conocimientos que cumplan con las expectativas personales, familiares e institucionales.

A las autoridades y estudiantes del plantel, que exijan el cumplimiento de las funciones y responsabilidades de orientación estudiantil, identificando las posibles causas y proponiendo las posibles soluciones, en especial, en casos de violencia intrafamiliar coordinando con las instituciones pertinentes.

Capacitar al personal encargado de estos procesos para que puedan llevar a cabo periódicamente jornadas de actualización y de salud mental escolar para todos, como estrategia para identificar la violencia como problema familiar que afecta al estudiante su vida personal y social.

\section{CONFLICTO DE INTERÉS}

Los autores declaran no tener conflicto de interés. 


\section{REFERENCIAS}

1. Colombia. Instituto Nacional de Salud. Biomédica: informe mundial sobre violencia y salud. Volumen 12. Bogotá. 2012.

2. Ferrer Lozano DM, Guevara DN, Lourdes, Martínez ME. La violencia como problema de salud. Miradas desde la realidad cubana. Gac Méd Espirit [Online]. Abril 2020: [citado 20 de marzo de 2021]. Disponible en: http:// scielo.sld.cu/scielo.php?script=sci_arttext\&pi$\mathrm{d}=\mathrm{S} 160889212020000100049 \&$ Ing=es.

3. González Leal EG, Pardo Gómez ME, Izquierdo JM. La violencia como problema de salud pública en Venezuela: algunas reflexiones desde la perspectiva del profesional de seguridad ciudadana. MEDISAN [Online]. 2017 Mayo [citado 2021 Mar 20]; 21(5): 642-649. Disponible en: http://scielo.sld.cu/scielo.php?script=sci_arttext\&pi$\mathrm{d}=$ S102930192017000500020\&lng=es.

4. Organización Mundial de la Salud. Informe sobre la situación mundial de la prevención de la violencia [Internet]. [Consultado $2018 \mathrm{fe}-$ brero 10]. Disponible en: http://apps.who.int/ iris/ bitstream/10665/145089/1/WHO_NMH_ NVI_14.2_spa.pdf.

5. UNICEF. La Violencia le hace mal a la familia. [Internet] 2017. [consultado 2018 febrero 10] p.3. Disponible en: http://metabase.uaem. $\mathrm{mx} / /$ handle/123456789/2596

6. López G, Lozano M. La violencia familiar. Situación actual y recomendaciones para su prevención. [Internet]. Iquitos, Perú. 2017. [Consultado 2018 febrero 10]. Disponible en: http://fadvamerica.org/wp-content/ uploads/2017/07/La-Violencia-Familiar-enlquitos-FADV.pdf.

7. Colombia. Gobernación de Córdoba. Secretaria del Interior y participación ciudadana. Observatorio del delito, informe marzo 2017. [Internet]. [Consultado 2018 febrero 24]. Disponible en: file:///C:/Users/Windows $\% 2010 /$ Desktop/TESIS\%20ARTICULOS/observatorio_delito_2017-03.pdf.

8. Vega-Lara L, Reales-Agón O. La violencia intrafamiliar y el aprendizaje en la escuela: un estudio etnográfico en la Institución Educativa Distrital (I.E.D) San Francisco Javier. [In- ternet]. Santa Marta: Maestría. Universidad del Magdalena. SUE Caribe; 2009. [Consultado 2018 febrero 24]. Disponible en: http:// repositorio.unimagdalena.edu.co/jspui/bitstream/123456789/1707/1/ME-00006.pdf.

9. Rivadeneira F. Violencia Intrafamiliar y sus efectos en el rendimiento académicos de los estudiantes de bachillerato del Instituto Vicente León de Latacunga. 2010 - 2011. Maestría. Universidad de Guayaquil; 2012, p.29.

10. Carrillo K, Menjivar K, Ortiz F. Violencia familiar y su influencia en el bajo rendimiento académico de adolescentes, resultado de un estudio cualitativo en San Salvador.; [Internet]. El Salvador: Doctorado. Universidad de El Salvador; 2015. p.20. [Consultado 2018 febrero 24]. Disponible en: http://ri.ues.edu.sv/ id/eprint/8596/

11. Delgado-Vásquez, VC. Violencia familiar y rendimiento académico de los alumnos de educación secundaria de la IE Felipe Humberto Tiravanty. [Internet]. El Verde-Chota: Cajamarca: 2014. p.12. [Consultado 2018 febrero 24]. Disponible en: http://repositorio.unc.edu. pe/handle/UNC/57

12. López-Mero P, Barreto-Pico A, Mendoza-Rodríguez ER, Salto-Bello MWA. Bajo rendimiento académico en estudiantes y disfuncionalidad familiar / Low academic result in students and dysfunctional families. [Internet]. 2015 Sep. [Citado 2018 mayo 19]; Medisan; 19(9):1163-1166, set.-set. 2015. Disponible en: https://pesquisa.bvsalud.org/portal/resource/pt/lil-760148.

13. Ministerio de Salud. Resolución 8430 de 1993. p.2-3. [Internet]. [Citado 2018 abril 04] Disponible en: https://www.minsalud.gov.co/ sites/ rid/Lists/BibliotecaDigital/RIDE/DE/DIJ/ RESOLUCION-8430-DE-1993.pdf.

14. Gonzaga-Gomero M. Violencia familiar y el rendimiento académico en el área de comunicación de los alumnos de 1er Grado de Secundaria en la Institución Educativa Domingo Mandamiento Sipán Hualmay - 2014. [Internet]. Perú: Maestría. Universidad César Vallejo; 2017. p.88. [Citado 2018 febrero 24]. Disponible en: http:// repositorio.ucv.edu.pe/ handle/UCV/5957 
15. Berrocal-Narváez N, Hanna-Lavalle $M$, Petro-Falón $\mathrm{L}$, et al. Accesibilidad a servicios preventivos en salud de jóvenes monterianos. Rev Avances en Salud. 2019;(3)2:33-41. DOI:10.21897/25394622.1745.

16. Cano M. Aproximaciones hacia una definición de bajo rendimiento escolar. [Internet]. Manizales: Maestría. Universidad de Manizales; 2016. p.4. [Citado 2018 febrero 24]. Disponible en: http://ridum.umanizales.edu. co:8080/xmlui/bitstream/handle/6789/2613/ Monsalve_Henry_De_Jes\%C3\%BAs_2016. pdf?sequence $=1$

17. Justo CA. Violencia intrafamiliar y la relación con el rendimiento académico de los estudiantes de la unidad educativa la Asunción de la parroquia la Asunción. periodo 2014-2015. [Internet]. Cantón Chimbo Provincia Bolívar: Maestría. Universidad Estatal de Bolívar; 2015. p.54. [Citado 2018 febrero 24]. Disponible en: http://190.15.128.197/bitstream/123456789/2158/1/TESIS\%20COMPLETA\%20NAPO\%20CALIZ.pdf

18. Canto J, Obregón JN. Violencia familiar y el rendimiento académico en el área de comunicación de los estudiantes del $1^{\circ}$ de secundaria, Delceba 34044 - Acostambo. [Internet]. Huancavelica: Perú; 2018. p.56. [Citado 2018 febrero 24]. Disponible en: http://repositorio. unh.edu.pe/handle/UNH/1759

19. Cabanillas-Zambrano C, Torres-Yajahuanca O. Influencia de la violencia intrafamiliar en el rendimiento académico en adolescentes de la Institución Educativa Fanny Abanto Calle, 2012. [Internet]. Mogrovejo: Perú. Pregrado. Universidad Católica Santo Toribio de Mogrovejo; 2013. p.43. [Citado 2018 febrero 24]. Disponible en: http://tesis.usat.edu.pe/xmlui/ handle/20.500.12423/389.

20. Alarcón-Alvarado ZA, Tufiño-Rivero N. Análisis del maltrato infantil reincidente de los casos que se presentan en la Defensoría de la niñez y adolescencia de la zona sur y la aplicación de la normativa. [Internet]. La Paz - Bolivia: Universidad Mayor de San Andrés. Facultad de Derecho y Ciencias Políticas. Carrera de Derecho; 2013. [Citado 2018 febrero 24]. Disponible en: https://repositorio.umsa. bo/handle/123456789/22540. 